\title{
Study of Cofactors Associated with Precancerous High-Grade Cervical Lesions at the Teaching Hospital Gabriel Touré, 2010 to 2015
}

\author{
Fané Seydou*, Bocoum Amadou, Sissoko Abdoulaye, Traoré Soumana Oumar, \\ Sanogo Siaka Amara, Mamadou Sima, Kouma Aminata, Traoré Alassane, \\ Tegueté Ibrahima, Traoré Youssouf, Mounkoro Niani
}

Teaching Hospital Gabriel Toure, Bamako, Mali

Email: *seydoufane@yahoo.fr

How to cite this paper: Seydou, F., Amadou, B., Abdoulaye, S., Oumar, T.S., Amara, S.S., Sima, M., Aminata, K., Alassane, T., Ibrahima, T., Youssouf, T. and Niani, M. (2019) Study of Cofactors Associated with Precancerous High-Grade Cervical Lesions at the Teaching Hospital Gabriel Touré, 2010 to 2015. Surgical Science, 10, 328-337.

https://doi.org/10.4236/ss.2019.109036

Received: August 27, 2019

Accepted: September 22, 2019

Published: September 25, 2019

Copyright $\odot 2019$ by author(s) and Scientific Research Publishing Inc. This work is licensed under the Creative Commons Attribution International License (CC BY 4.0).

http://creativecommons.org/licenses/by/4.0/ (c) () Open Access

\begin{abstract}
Introduction: High grade dysplasia of the cervix has a high incidence and can progress to cervical cancer. The aim was to study cofactors associated with high-grade cervical dysplasia. Methodology: This was a retrospective case-control study without matching. Women with high grade dysplasia were the cases while those with a normal screening test represented the controls. The study took place at the Gabriel Touré University Hospital Center in Bamako. We included 351 cases and 420 controls. The capture and analysis were performed using the SPSS 20 software. A univariate and multivariate logistic regression analysis was performed for the analysis of risk cofactors. The statistical tests used were the odds ratio and its confidence interval and the statistical significance threshold was set at $\mathrm{p}<0.05$. Results: In univariate analysis, the co-factors statistically significantly associated with the occurrence of high-grade dysplasia were parity $0.6(0.5-0.9)$, gestational $0.7(0.5-0.9)$, smoking of the spouse $3.4(1.1-11.3)$, the non-schooling $1.4(1.2-2.1)$. In multivariate analysis after adjusting for confounding factors, two co-factors have significantly increased the risk of high-grade dysplasia: lack of schooling $1.4(1.2-2.0)$ and polygamy $1.5(1.4-2.5)$. Conclusion: At the end of this study, polygamy and lack of schooling were the main risk factors. The prevention of cervical cancer will go through the education of girls and women as well as communication for behavioral change and social change.
\end{abstract}

\section{Keywords}

High Grade Dysplasia, Cofactor, Cervical Cancer, Teaching Hospital, Gabriel Touré 


\section{Introduction}

Precancerous high-grade lesions or high-grade dysplasia of the cervix constitute the disorganization of the architecture of the squamous epithelium with loss of normal maturation and stratification, cytonuclear atypia and mitosis reaching the middle third and superior to the epithelium and respect the basement membrane [1] [2]. High-grade dysplasia consists of intraepithelial neoplasia of the second-degree cervix (CIN2) and intraepithelial neoplasia of the third-degree cervix. (CIN3). Low grade dysplasia is composed of first-degree cervical intraepithelial neoplasia (CIN1). High grade dysplasia and cervical cancer are non-transmissible pathology of infectious origin [3] [4]. Persistent infection of high-risk human papillomavirus (HPV) oncogene is considered the main but not sufficient cause alone for causing high-grade dysplasia and cervical cancer [3] [5]. These diseases have been recognized by the World Health Organization (WHO) as being $100 \%$ attributable to HPV infection [6]. The natural history of cervical cancer occurs along the continuum of precancerous histological lesions or cervical intraepithelial neoplasia (CIN). These follow the persistence of infection with high-risk oncogenic HPV for each grade of the precancerous cervical lesion (CIN1, CIN2, CIN3), there is a probability of regression that varies by $57 \% ; 43 \% ; 32 \%$. In addition, the probability of persistence of CIN1, CIN2, CIN3 is $32 \% ; 35 \% ; 56 \%$. In fact, the probability of progression of CIN1, CIN2, CIN3 towards invasive cancer is also $1 \%$; $5 \%$; and greater than $12 \%$ [3] [7] [8]. High grade dysplasia and cervical cancer pose a major public health problem because of their high incidence and high mortality from cervical cancer. The specific role of cofactors in the development of high-grade dysplasia and cervical cancer is not fully understood [3] [5]. This scientific deficit in the carcinogenesis of cervical cancer motivated us to undertake the present study in Mali where resources are limited and the burden of cervical cancer is enormous [6]. Previous studies in Mali on cervical cancer are mainly descriptive because of the scarce resources allocated to research and the poor quality of existing data in our structure's health. It is in this context that we initiated this work to study cofactors associated with precancerous high-grade cervical lesions.

The specific objectives of this study were:

- describe the general characteristics of cases and controls,

- compare the characteristics of participants with high grade dysplasia to those with no dysplasia or cervical cancer.

To clarify the influence of different cofactors we asked the following research question: Are women with high grade dysplasia more risk cofactors than those free of dysplasia?

The objectives of this study will be achieved by verifying the following research hypothesis: Cofactors increase the risk of high-grade cervical dysplasia.

\section{Material and Methods}

\subsection{Study Framework}

This study was conducted at Gabriel Touré Hospital Center in Bamako in the 
cervical cancer screening and cervical pathology unit.

\subsection{Type and Period of Study}

We performed an unpaired case-control study. Data collected included women who visited between January 1, 2010 and October 31, 2015.

\subsection{Study Population}

It consisted of all sexually active women who received cervical cancer screening during the study period. These were referred or evacuated women from the district of Bamako or from the interior of the country. They were polygamous out of school and housewives in the majority of cases. These were women who were not informed about cervical cancer. Since 2003 the treatment of all precancerous and cancerous cervical lesions has been centralized at Gabriel Touré Teaching Hospital in Bamako. A monthly meeting is organized every last Thursday of the month and brings together all providers involved in cervical cancer screening at various levels of the Bamako District health system. This meeting takes stock of the number of women screened, the number of lesions detected and their management by center as well as their follow-up.

\subsection{Sampling}

Were included in this study all women who met the following criteria:

- Inclusion Criteria: Included in this study were all women who met the following criteria:

- sexually active woman who has been screened for cancer of the cervix;

- free from dysplasia or cervical cancer confirmed by histology;

- woman with high grade dysplasia confirmed histology;

- woman having a record exploitable.

\section{- Exclusion criteria}

- Woman having a total hysterectomy;

- Virgin woman;

- Woman with cervical cancer confirmed by histology;

- Woman whose histology was not performed;

- Woman with an unexplainable record.

- Sampling technique: Cases and controls were defined as follows:

Definition of cases: it included any sexually active woman whose biopsy has shown a high-grade cervical lesion was a case.

Definition of controls: it included any sexually active woman whose histology has not confirmed any dysplasia or cervical cancer.

\section{- Minimal height of sampling}

A full sampling was conducted from 2010 to 2015. We have defined certain values to make the calculation of the minimal height of the sample. The number of controls was given in a study conducted by Bayo et al. [9] in 2002 in Bamako which found $14 \%$ of large multiparty with witnesses (one case for one control). 
The power was $90 \%$ and the level of confidence was $95 \%$. The calculation of the minimal height of the sample was made by a software called Epi info. Given the number of cofactors to study the parity was chosen for this calculation. We got at least 200 high grade dysplasia for at least 200 controls, by taking out $10 \%$ as loss rate 440 cases were found.

\section{Analysis and data processing strategy:}

The data analysis and processing was made by SPSS version 20 software. The cases and the witnesses were separated during this process. The data were analyzed to make sure of the speed of distribution. We conducted a univariate and multivariate study. The following statistical tests were used during the univariate analysis. The non-parametric comparison test of two quality variables (Khi-deux) for the comparison of proportions if the expected number was less than 5, the exact test of Fisher if the number is less than 5, the student's test and the analysis of Anova (variance) for the comparison of averages. The logistic regression was used including all the variables in literature with one P less or equals $10 \%$ and the other variables with one $\mathrm{p}$ less or equals 5\%. Adjusted odds Ratio and their confidence interval (IC) at 90 were calculated to take into account this third cofactor. This combination was considered significant if the confidence interval excluded 1 . We used the step-by-step logistic regression method with $5 \%$ input and filtered at $10 \%$ until the final model was obtained.

\section{Results}

\subsection{Descriptive Epidemiology}

We collected 351 women with dysplasia of high and 420 subjects free from dysplasia or cancer. Women with high grade dysplasia had a middle and median age of 37 with a minimum age of 18 and a maximum age of 73 years. Women with dysplasia or cancer had an average age of 39 years and a median age of 40 with extremes ranging from 18 to 75 years. The difference between the mean age of cases and the average age of control was significant $(t=2.034, \mathrm{p}=0.042)$. HIV-positive women were $6.3 \%$ for cases and $3.6 \%$ for controls. There were $32.2 \%$ large multigesty in women with high grade dysplasia and $40.7 \%$ in dysplasia free women. Large multiparous cases accounted for $24.8 \%$ and $33.3 \%$ of women with dysplasia or cancer. Oral contraception was used in $52.1 \%$ of women with high grade dysplasia and $48.6 \%$ of patients without dysplasia or cancer. Indeed, $55.8 \%$ of women with high grade dysplasia were housewives compared to $69.0 \%$ of those who were free from dysplasia or cancer. We found $48.4 \%$ of polygamy in women with high grade dysplasia and $45 \%$ in free from dysplasia or cancer. Illiteracy accounted for $69 \%$ of those with high grade dysplasia and $61 \%$ of those without dysplasia or cancer. Women who had had their first sexual intercourse before the age of 18 accounted for $58.1 \%$ of women with high grade dysplasia and $63 \%$ of those who were free from dysplasia or cancer. Women who had their first pregnancy before 18 years of age accounted for $46.2 \%$ of patients with high grade dysplasia and $47.7 \%$ of patients with dysplasia or cancer. In fact, married 
women accounted for $87.7 \%$ of women with high grade dysplasia and $89.6 \%$ of those who were free from dysplasia or cancer. In this study we determined the distribution of HPV strains in both groups (Table 1).

We also specified the distribution of the bacteria found in PV in both groups (Table 2).

We screened 12,623 women among whom were collected 351 cases and 420 controls for a period of 6 years as shown in the flowchart (Figure 1).

The distribution of precancerous lesions of the cervix depending on age is shown in Figure 2.

The 30 - 39 age group was more concerned with precancerous high-grade and low-grade cervical lesions. However, adolescents (age $\leq 19$ years) were poorly affected by these precancerous lesions (Figure 2). The extreme ages of precancerous

Table 1. Distribution of HPV strains found in cases and controls.

\begin{tabular}{ccc}
\hline HPV & \% Cases $(\mathbf{n}=351)$ & \% Controls $(\mathbf{n}=420)$ \\
\hline HPV16 & $8.2 \%$ & $1.2 \%$ \\
HPV18 & $4.2 \%$ & $2.5 \%$ \\
Other high risk HPV & $12.3 \%$ & $10 \%$ \\
\hline
\end{tabular}

$\mathrm{HPV}=$ Human papillomavirus.

Table 2. Distribution of germs found during vaginal sampling in cases and controls.

\begin{tabular}{ccc}
\hline Germs & \% Cases $(\mathbf{n}=\mathbf{3 5 1})$ & \% Controls $(\mathbf{n}=\mathbf{4 2 0})$ \\
\hline Chlamydiae & 1.5 & 0.7 \\
Ureaplasma & 7.3 & 3.5 \\
Gardnerella & 10.2 & 9.3 \\
Trichomonas vaginalis & 23.4 & 1 \\
Mycoplasma & 2 & 14.2 \\
Candida albicans & 31.2 &
\end{tabular}

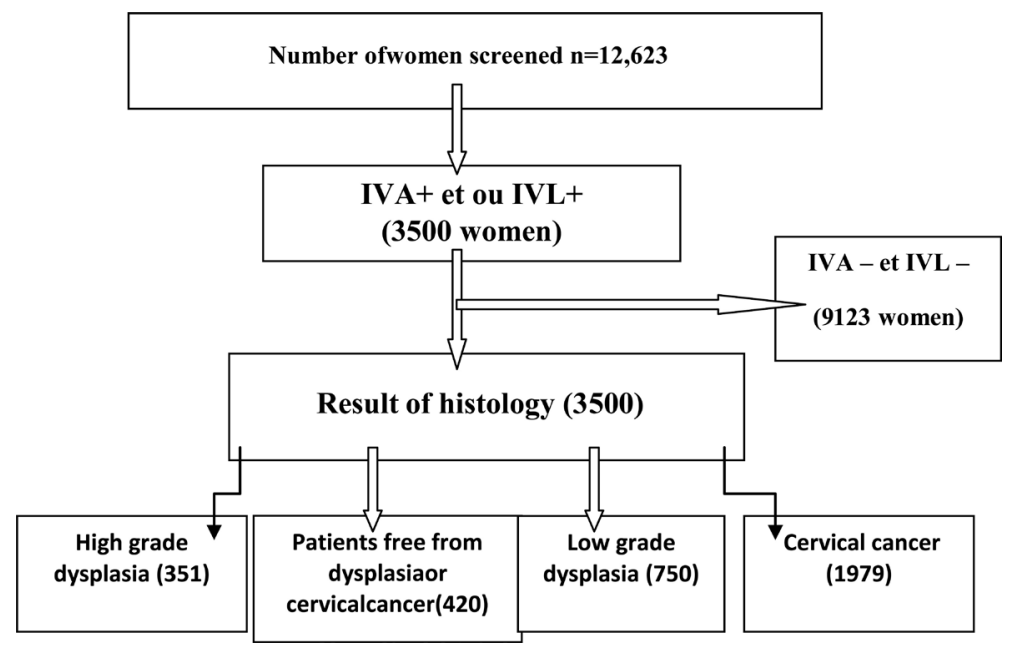

Figure 1. Inclusion flowchart of cases and controls. 


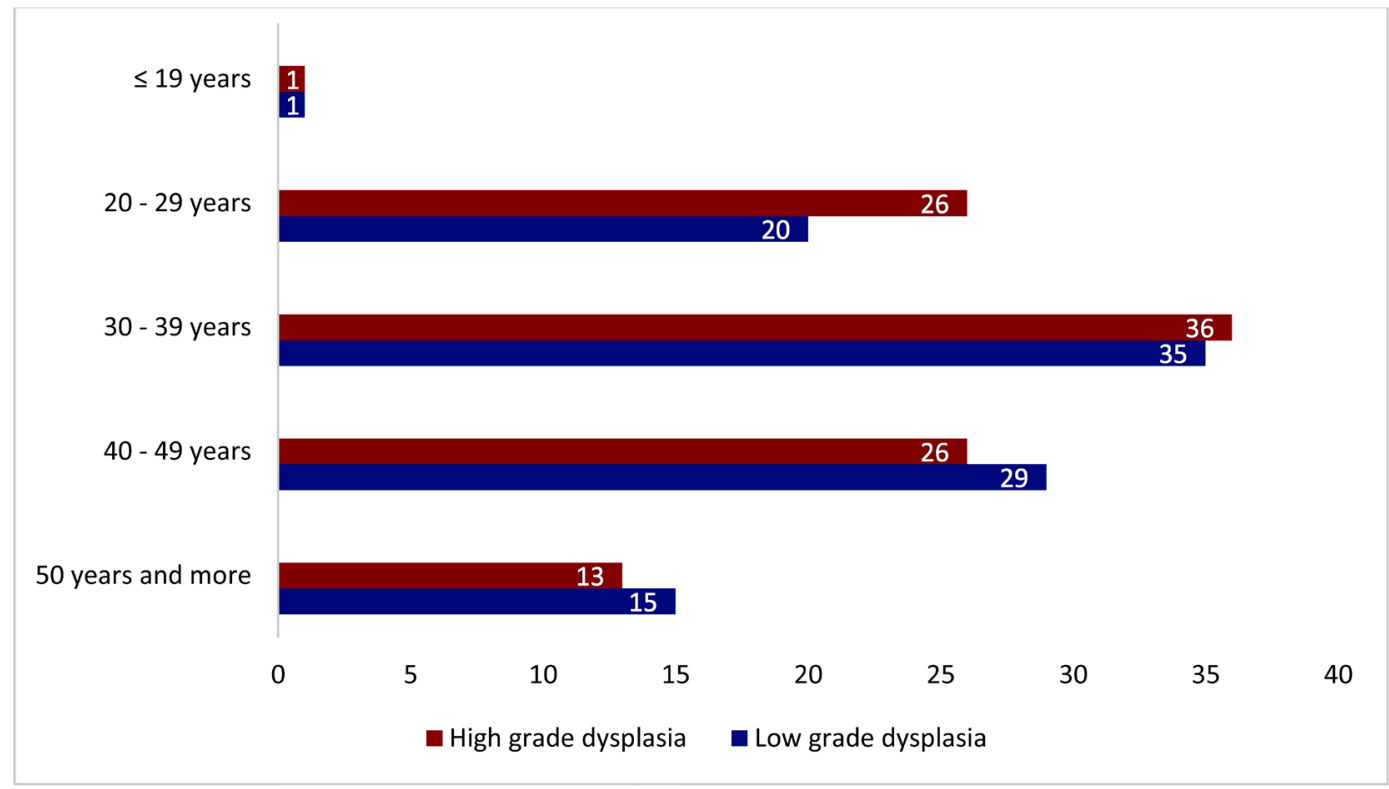

Figure 2. Precancerous lesions of the cervix depending on age.

high-grade lesions were 18 and 73 years old and those of low-grade dysplasia were 16 and 75 years old.

\subsection{Analytical Epidemiology}

\section{Average age of women with high grade dysplasia}

We compared the average age of women with high grade dysplasia to those free from dysplasia or cancer. In fact, the average age of women with high grade dysplasia was $37 \pm 0.5$ years, while that of patients without dysplasia was $39 \pm 0.5$ years. The difference between these two average ages was significant $(t=2.034, p$ $=0.042$ ). The analysis of the variance confirmed the results of the test. It showed that the average age of patients with high grade dysplasia was statistically different from that of free from dysplasia or cancer (Anova $\mathrm{F}=3.330, \mathrm{p}=0.036$ ).

\section{Risk factors}

The risk factors have been studied in Table 3 below.

\section{Discussion and Comments}

In the light of the work of the literature, the main results of this work have been commented upon and discussed.

\subsection{Limitations of the Study}

This study presented some methodological limitations. As the data collection was retrospective, not all independent variables could be fully informed. Indeed, all women with IVA and or IVL negative were excluded because it is not ethical to practice biopsy on a normal cervix. However, these women with IVA negative and or IVL negative could be real witnesses. Pathological examination was selected as a reference test for the diagnosis of high-grade dysplasia. This was an 
Table 3. Comparison of the characteristics of the participants in the study.

\begin{tabular}{|c|c|c|c|c|}
\hline Cofactors & univariate Analysis OR & $\mathrm{p}$ & $\begin{array}{l}\text { Multivariate Analysis } \\
\text { OR }_{\text {ajusted CI } 95 \%}\end{array}$ & $\mathrm{p}$ \\
\hline \multicolumn{5}{|l|}{ Age (years) } \\
\hline $15-49$ & 1 (Ref) & & 1 (Ref) & \\
\hline$\geq 50$ & $0.9(0.6-1.3)$ & 0.6 & $0.9(0.6-1.3)$ & 0.6 \\
\hline \multicolumn{5}{|l|}{ Parity } \\
\hline$<6$ & 1 (Ref) & & 1 (Ref) & \\
\hline$\geq 6$ & $0.6(0.5-0.9)$ & 0.3 & $0.8(0.4-1.4)$ & 0.3 \\
\hline \multicolumn{5}{|l|}{ Gestity } \\
\hline$<6$ & 1 (Ref) & $<6$ & 1 (Ref) & \\
\hline$\geq 6$ & $0.7(0.5-0.9)$ & 0.015 & $0.8(0.4-1.5)$ & 0.6 \\
\hline \multicolumn{5}{|c|}{ Schooling attendance } \\
\hline Yes & $1.4(1.1-2.0)$ & 0.02 & $1.4(1.2-2.0)$ & 0.04 \\
\hline No & 1 (Ref) & & 1 (Ref) & \\
\hline \multicolumn{5}{|l|}{ Marital status } \\
\hline Monogamy & 1 (Ref) & & 1 (Ref) & \\
\hline Polygamy & $1.2(0.8-1.5)$ & 0.341 & $1.4(1.2-2.5)$ & 0.04 \\
\hline
\end{tabular}

analytical study to identify associated factors contributing to precancerous lesions. Understanding the role of co-factors would enable field health workers to better develop key messages for the population. This work attempts to explain the recent epidemiology of high-grade dysplasia and cervical cancer which is the rejuvenation of patients with these conditions. This study will prevent cancer of the cervix.

\subsection{Prevalence of High-Grade Precancerous Lesions}

We collected 351 cases of high-grade dysplasia on 12,623 women who had cervical cancer screening, a prevalence of high-grade dysplasia of $2.8 \%$ (Figure 1). This prevalence is superior to that of Traore, S. et al. in 2003 [10] who found $2.2 \%$ at the beginning of cervical cancer screening in Mali. The prevalence of dysplasia of $4.3 \%, 15.6 \%$ and $11.7 \%$ respectively reported in Kenya, Zimbabwe and Ivory Coast [11].

\subsection{Average Age of Women with High Grade Dysplasia}

The average age of women with high grade dysplasia was $37 \pm 0.5$ years compared to $39 \pm 0.5$ years for free from dysplasia or cancer. These age averages are lower than those of Bayo et al. [9] who found 46 years for cases and controls. Khaoula, B. et al. [11] found an average of $46 \pm 8.7$ years for high grade dysplasia in a descriptive study in Morocco. The epidemiology of precancerous and cancerous lesions is being modified. Indeed, the average age of CIN was 30 years 
and 45 years for invasive cancer. Today we notice a rejuvenation of the average age of precancerous and cancerous lesions in the general population. The appearance of high-grade cervical lesions and cancer of the cervix at an earlier age seems to be linked to the upsurge of risk factors such as poor socioeconomic conditions, early intercourse, multiple sexual partners, exposing to human papilloma virus (HPV), sexually transmitted infections and finally the large multiparity decreased immunity plays also an important role in the rejuvenation of women with invasive cervical cancer. For Dargent, the relative increase in the frequency of juvenile forms of cervical cancer has been observed in all reference institutes. Mainan joins Dargent in stating that cervical cancer in young women is explained by the fact that HIV infection in young people is associated with a prevalence of severe dysplasia of the cervix and a more severe evolution of lesions than the immune deficiency is deep [11].

\subsection{Marital Status}

In our study, polygamous women with spouses with more than two wives were 1.4 times more likely to have a high-grade injury compared to monogamous women $[$ OR95\% = $1.4(1.1-1.8)$ ] (Table 3$)$. This finding duplicates data from the literature that found that polygamy with more than 4 partners would be a risk factor. A case-control study by Bayo, S. et al. in 2002 found that polygamy with a spouse with more than 2 wives was a risk factor for cervical cancer OR5\% $=5.3(1.3$ - 21.3) [9] [12]. A study by Tegueté et al. [12] confirmed that the risk of a precancerous lesion increased 3.1 times when the marriage regime was polygamous. This polygamy is a common legal practice in Mali where the man can marry up to four women. The number of sexual partners increases the risk of being reached by high-risk oncogenic HPV [9] [13].

\subsection{Schooling Attendance}

Out-of-school women were 1.4 times more likely to develop a high-grade cervical lesion [ORIC95\% = $1.4(1.1$ - 2)] compared to women in school. This difference was statistically significant (Table 3 ). A study conducted by Antoine Jacquet et al. 2002 [7] found that women in school had 50\% protection compared to those who were out of school with ORIC95\% $=0.5(0.3-0.6)$ [7] [9] [10] [14]. The risk of developing high-grade cervical lesion by these out-of-school women is related to under-reporting and lack of cervical cancer screening. These reasons make oncogenic HPV infection become common and persistent. Low levels of knowledge and perception in the general population have been reported up to $60 \%$ of women had never heard from about cervical cancer and only $7.8 \%$ had heard about it.

\section{Conclusion}

Effective prevention of cervical cancer requires active research, screening, and, where appropriate, management of the co-wives of women with high-grade cer- 
vical lesions. Information, education, communication for the health of the population, prophylactic vaccination against HPV and the schooling of young girls are essential. It is necessary to put in place an effective action plan against cervical cancer.

\section{Conflicts of Interest}

The authors declare no conflicts of interest regarding the publication of this paper.

\section{References}

[1] Lansac, J., Lecomte, P. and Marret, H. (2007) Gynecology for the Practitioner: Cervical Cancer. Masson, Paris, 85-94.

[2] Komongui, D.G., Adjahoto, I.O., Tonato, B.A., Teguete, I. and Perrin, R.X. (2006) Feasibility Study and Organizational Aspect of Cervical Cancer Screening by Visual Inspection with Acetic Acid and Lugol, in Cotonou, Benin. Journal of SAGO, 7, 31-36

[3] Duport, N., Heard, I., Barré, S. and Woronoff, A.S. (2014) Cervical Cancer: State of Knowledge in 2014. Bulletin Epidemiologique Hebdomadaire, 2014, 220-221.

[4] Okonofua, F. (2015) Prevention and Control of Cervical Cancer in Africa: A Call to Action. African Journal of Reproductive Health, 19, 12-16

[5] Duport, N. (2008) Epidemiological Data on Cervical Cancer: State of Knowledge. VS, 1-31.

[6] Zanier, K., Sidi, O.A., Boulade, L.C., Rybin, V., Chapel, A., Atkinson, A., et al. (2012) Solution Structure Analysis of the HPV16 E6 Oncoprotein Reveals to Self-Association Mechanism Requires for E6-Mediated Degradation of p53. Structure, 20, 604-617. https://doi.org/10.1016/j.str.2012.02.001

[7] Walboomers, J.M., Jacobs, M.V., Manos, M.M., Bosch, F.X., Kummer, J.A., Shah, K.V., et al. (1999) Human Papillomavirus Is a Necessary Cause of Invasive Cervical Cancer Worldwide. The Journal of Pathology, 189, 12-19. https://doi.org/10.1002/(SICI)1096-9896(199909)189:1<12::AID-PATH431>3.0.CO; $\underline{2-\mathrm{F}}$

[8] Jaquet, A., Odutola, M., Ekouevi, K.D., Tanon, A., Ogo, E., Akakpo, J., et al. (2015) Cancer and HIV Infection in Referral Hospitals from Four West African Countries. Cancer Epidemiology, 39, 1060-1065. https://doi.org/10.1016/j.canep.2015.09.002

[9] Bayo, S., Bosch, F.X., Sanjose, S., Muñoz, N., Combita, A.L. and Coursaget, P. (2002) Risk Factors of Invasive Cervical Cancer in Mali. International Journal of Epidemiology, 31, 202-209. https://doi.org/10.1093/ije/31.1.202

[10] Serdouma, E., Koffi, B., Konzelo, N., Heredobona, L.S., Dapka, S. and Rawako, D.B. (2010) Prevalence of Precancerous Lesions of the Cervix in Four Cities of the Central African Republic.

[11] Dargent, D., Kouakou, F. and Cholmier, M. (1991) Cervical Cancer in Women Under 35 Years and under: A New Disease. Reviews in Obstetrics and Gynecology, 86, 635-638.

[12] Tegueté, I., Thera, A. and Traore, Y. (2015) Epidemiology of Cervical Uterine Infection with Oncogenic Papillomavirus at the Gabriel Touré Teaching Hospital, Bamako. In: Hedon, B. and Deruelle, P., Eds., Updates in Gynecology and Obstetrics, CNGOF, 447-458. 
[13] Franco, E.L., Schlecht, N.F. and Saslow, D. (2003) The Epidemiology of Cervical Cancer. The Cancer Journal, 9, 348-359.

https://doi.org/10.1097/00130404-200309000-00004

[14] Kabibou, S., Luc, B., Achille, O. and Xavier, R.P. (2015) Factors Associated with Precancerous and Cancerous Cervical Lesions in the City of Parakou in Benin. European Scientific Journal, 11, 178-183. 